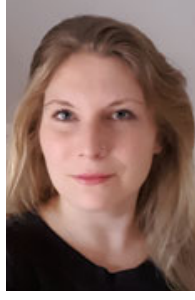

Michaela Wenzel

2013 Promotion in Biologie an der Universität Bochum über Antibiotika-Stressantworten. 2014-2017 Postdoc an der Universität von Amsterdam, Niederlande. 2017-2018 PostdocStipendium des Amsterdam Infection and Immunity Institute, Amsterdam University Medical Centers. Seit 2019 Assistant Professor an der Chalmers University of Technology in Göteborg, Schweden.

DOI: $10.1007 / \mathrm{s} 12268-019-0220-1$

(c) Springer-Verlag 2019

Die dramatische Zunahme von Antibiotikaresistenzen und die noch immer zu niedrigen Zulassungszahlen neuer antimikrobieller Wirkstoffe haben unsere Gesellschaft in eine handfeste Antibiotikakrise geführt. Um aus dieser Sackgasse wieder herauszufinden, müssen dringend neue Wirkstoffe entwickelt werden. Zur Vermeidung einer schnellen Resistenzentwicklung sind Substanzen ideal, die kein spezifisches Bakterienprotein inhibieren, sondern strukturelle Ziele angreifen oder mehr als eine Zielstruktur hemmen. Dies ist insbesondere bei Membran-angreifenden Antibiotika der Fall.

Lange Zeit wurde angenommen, dass Substanzen wie Daptomycin die Funktion der Membran durch Porenbildung stören. Untersuchungen an Modellmembranen bestätigten dies. Neue Entwicklungen im Bereich zellbiologischer Methoden und hochauflösender Fluoreszenzmikroskopie erlauben jedoch heutzutage, diese Prozesse an lebenden Bakterien in Echtzeit zu verfolgen. Dies zeigte, dass die weitverbreiteten Porenmodelle zu einfach gedacht sind und die Situation in lebenden Zellen mit komplexen Membranen zumeist nicht treffend widerspiegeln können. Während einige Membran-angreifende Sub-

Nachwuchswissenschaftler/innen stellen sich vor

\title{
Membranaktive Antibiotika: neue Mechanismen gegen ein altbekanntes Ziel
}

\author{
MICHAELA WENZEL \\ DEPARTMENT OF BIOLOGY AND BIOLOGICAL ENGINEERING, CHALMERS UNIVERSITY \\ OF TECHNOLOGY, GÖTEBORG, SCHWEDEN
}

stanzen, beispielsweise die Tyrocidine, tatsächlich zu Poren in der Membran führen, wissen wir heute, dass dies mit weitreichenden zusätzlichen Membraneffekten und oftmals sekundären Zielstrukturen oder -prozessen verbunden ist. Des Weiteren werden immer mehr Wirkstoffe bekannt, die hoch komplexe, porenunabhängige Mechanismen der Membraninhibierung besitzen (Abb. 1).

Ein gutes Beispiel dafür ist Daptomycin, das lange selbst als Porenbildner galt. Mithilfe neuer in vivo-Methoden konnten wir jedoch zeigen, dass Daptomycin keine Poren bildet, sondern bevorzugt an fluide Membrandomänen bindet. Dabei stört es deren Organisation derart, dass dort lokalisierte, periphere Membranproteine von der Membran abfallen. Die in diesen Domänen ablaufende laterale Zellwandsynthese ist daher stark betroffen [1]. Einen weitreichenderen Effekt hat das antimikrobielle Peptid cWFW. Wie wir zeigen konnten, verursacht es eine Phasentrennung der Membran in fluide und rigide Domänen. Dies hat eine Trennung von peripheren und integralen Membranproteinen zur Folge, wodurch Proteinkomplexe getrennt und somit wirksam gehemmt werden [2]. Ein weiteres interessantes Beispiel ist Rhodomyrton, ein pflanzlicher Wirkstoff aus der traditionellen asiatischen Medizin. Dieses natürliche Antibiotikum biegt die Membran nach innen und bildet so intrazelluläre Vesikel. Interessanterweise geht dies mit einer Erhöhung der Fluidität in diesen Bereichen einher, was eine dortige Anhäufung von Membranproteinen nach sich zieht. Diese werden dann in den Vesikeln eingeschlossen und somit inaktiviert [3].

Die genannten Beispiele stellen die besondere Wichtigkeit von Membranfluidität, -domänen und -proteinen heraus. Diese Faktoren haben bisher wenig Beachtung in der Antibiotikaforschung gefunden, auch weil die passenden Methoden fehlten, dies in vivo ausreichend zu untersuchen. In meiner Arbeitsgruppe untersuche ich das Zusammenspiel dieser Faktoren und ihre Bedeutung für Antibiotikamechanismen und -resistenzen mit zellbiologischen Methoden.

\section{Danksagung}

Ich danke Fredrik Westerlund und Stefan Hohmann für die Unterstützung beim Aufbau meiner Arbeitsgruppe.

\section{Literatur}

[1] Müller A, Wenzel M, Strahl H et al. (2016) Daptomycin inhibits bacterial cell envelope synthesis by interfering with fluid membrane microdomains. Proc Natl Acad Sci USA 113:E7077-E7086

[2] Scheinpflug K, Wenzel M, Krylova O et al. (2017) Antimicrobial peptide cWFW kills by combining lipid phase separation with autolysis. Sci Rep 7:44332

[3] Saeloh D, Tipmanee V, Jim KK et al. (2018) The novel antibiotic rhodomyrtone traps membrane proteins in vesicles with increased fluidity. PLoS Pathog 14:e1006876

\section{Korrespondenzadresse:}

Dr. Michaela Wenzel

Division of Chemical Biology

Department of Biology and Biological Engineering

Chalmers University of Technology

Kemivägen 10

SE-41296 Göteborg

wenzelm@chalmers.se

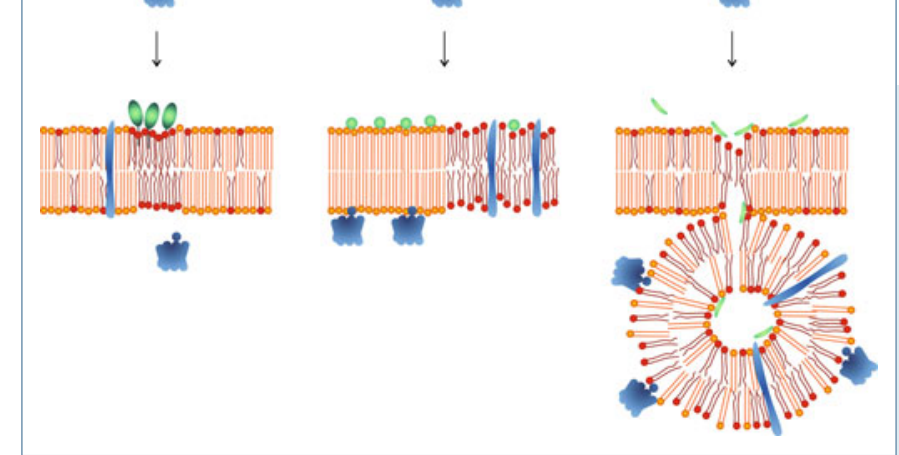

Abb. 1: Porenunabhängige Mechanismen der Membraninhibierung. Daptomycin bindet an fluide Membrandomänen und packt fluidisierende Lipide in dichte Domänen, wodurch periphere Membranproteine abfallen. cWFW trennt die Membran in rigide und fluide Bereiche, wodurch periphere und membrandurchspannende Proteine getrennt werden. Rhodomyrton biegt die Membran an fluiden Domänen nach innen und bildet so Vesikel, in denen Membranproteine eingeschlossen werden. Rot: fluidisierende Lipide (ungesättigte, kurze oder unverzweigte Fettsäuren); orange: rigidisierende (nicht-fluidisierende) Lipide (gesättigte, lange oder verzweigte Fettsäuren); blau: Membranproteine, grün: Antibiotika. 